\title{
Cycloid flows induced by the Large horizontal coherent structures in the vegetated compound channel.
}

\author{
Truong Hong Son ${ }^{1,2, *}$ and WSJ Uijttewaal ${ }^{1, * *}$ \\ ${ }^{1}$ Hydraulic department,Faculty of Civil Engineering and Geosciences, Delft University of Technology, \\ Delft, The Netherlands \\ ${ }^{2}$ Faculty of Civil Engineering, Thuy Loi University, Hanoi, Vietnam.
}

\begin{abstract}
Vegetation in general and mangrove, in particular, plays a significant role in the protection of the coastal and estuarine regions from erosion. In particular, estuarine mangrove forests can efficiently reduce the impact of a strong along-bank flow during high tides and high river discharge, creating shelter regions for the development of the ecological system. As estuarine mangrove is usually inundated during high tides and exposed to strong tidal flows, the hydrodynamic of estuarine mangrove forest is similar to that of a vegetated compound channel. In order to gain more insight into this field of the research, a hydraulic laboratory experiment of a shallow flow field in a vegetated compound channel has been conducted. Experimental results confirm a pronounced existence of large horizontal coherent structures (LHCSs). Although the presence of the large horizontal coherent structures in the vegetated channel has been well recognized, it is still unclear how they affect the flow field, mass and momentum exchange in the vegetated compound channel. Detailed analyses of the experimental data reveal more about the role of the large horizontal coherent structures. The flow field under the effect of the large coherent structures shows a spatially and temporally cycloid motion, contributing a large part to the momentum and mass transfer. The quadrant analysis of the Reynolds shear stresses has been applied to examine the connection between the motion of the large horizontal coherent structures and their associated cycloid flow events.
\end{abstract}

\section{Introduction}

The significant protecting role of vegetation in general and mangroves in particular for coastal and estuarine regions has been increasingly recognized. The vegetation not only absorbs penetrated waves but also efficiently reduces the impact of a strong along-bank flow during high tides and high river discharge, creating shelter regions for the sustainable development of the ecology. Their strong and dense stems, canopies and roots are important factors controlling the dynamics of the coastal line as well as the estuarine river bank. In the Mekong Delta Estuaries, mangroves usually dominate the floodplain regions with a gentle slope of about $1: 10$. Moreover,in many large mangrove forests, only a very narrow strip is left due to the

\footnotetext{
*e-mail: s.truonghong@tudelft.nl

**e-mail: W.S.J.Uijttewaal@tudelft.nl
} 
human impacts. The observed mangrove width of about 50 to $600 \mathrm{~m}$ is small compared to a river's main channel width of 1000 to $2000 \mathrm{~m}$. The river banks at those locations are usually eroding at a rate of two to four meters per year (Figure 1).

The degradation of the mangrove forest together with the accelerated eroded river banks has been studied in terms of the "squeeze" phenomenon [1, 2]. Because of an estuarine mangrove squeeze, the width of mangrove forest is usually very narrow, the presence of lateral tidal creeks is hardly observed and can be ignored. The main interaction is the lateral exchange through the mixing layer between the main river channel and the vegetated floodplain region. In this situation, the hydrodynamics of a mangrove forest is similar to that of a vegetated channel [2].

Numerous studies have examined the hydrodynamics of a compound channel, particularly focusing on the presence of large horizontal coherent flow structures (LHCSs) caused by the Kelvin-Helmholtz $(\mathrm{KH})$ instability formed at the interface of the slow and fast flowing regions [3-7]. However, understanding LHCSs is still limited, especially in cases with vegetation. For instance, it is still unclear how the LHCSs respond to changes of vegetation and how the LHCSs have influcences on the shallow flow field of a vegetated compound channel.

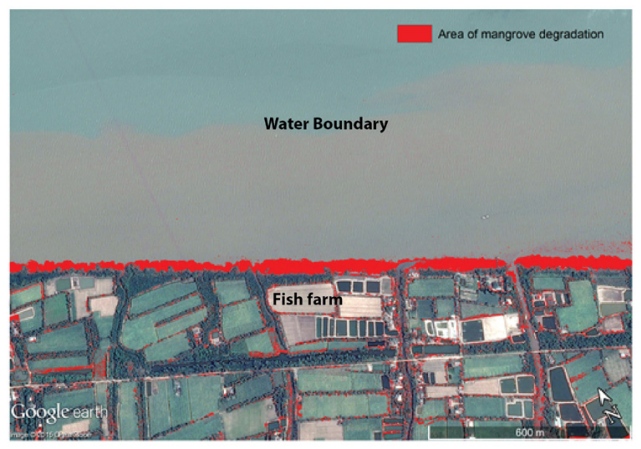

Figure 1. Mangrove degradation along a straight part of Dinh An estuary, An Thanh province, Vietnam. The red area illustrates the area loss of mangrove from 2006 to 2014. Fish farms constructed close to the water boundary push the mangrove into a narrow fringe zone. This area is suffering from erosion with a rate of about $2.5 \mathrm{~m} \mathrm{yr}^{-1}[2]$.

\section{Methodology}

In order to obtain more insight, a laboratory experiment of a shallow flow field in a vegetated compound channel was conducted in a flume of the Fluid Mechanics Laboratory at Delft University of Technology. A top view and a cross-section of the experimental setup can be seen in Figure 2. A Nortek Acoustic Doppler Velocity meter (ADV) was used to measure velocities at a sampling rate of $25 \mathrm{~Hz}$. Large Scale Particle Image Velocimetry (LSPIV) was also applied to measure the movement of the free surface. Sufficient contrast between the particles and the flume bottom can be achieved by using floating black polypropylene tracer particles $(d=2 \mathrm{~mm})$. A sampling frequency of $10 \mathrm{~Hz}$ was used in the present experiments. 

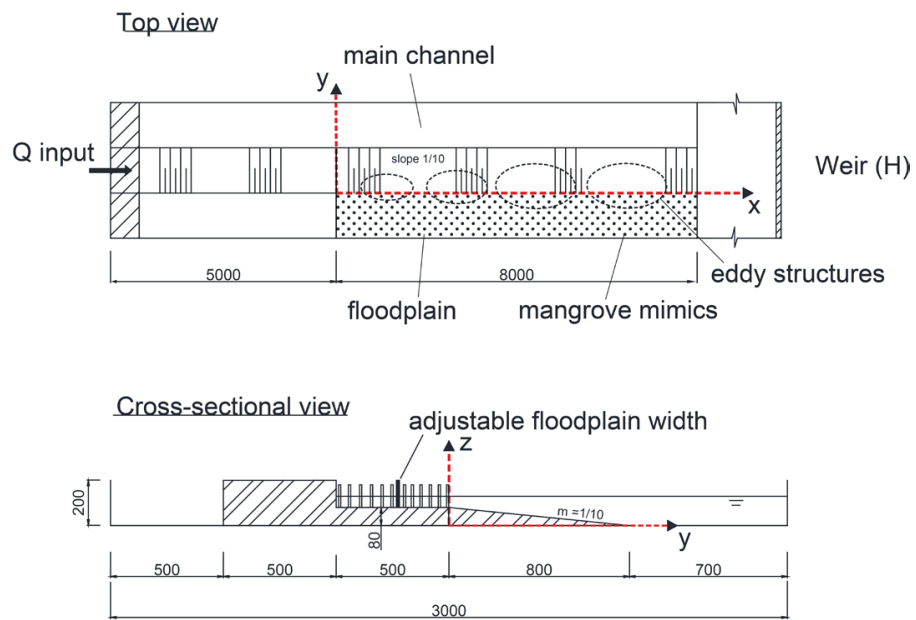

Figure 2. Schematic view of the experiment set up (units in $\mathrm{mm}$ ), the presence of large coherent structures in the flow field. Not to scale.

Table 1. Experiment scenarios

\begin{tabular}{llll}
\hline$\phi$ & $\begin{array}{l}\text { Density 1 } \\
\text { (no vegetation) }\end{array}$ & $\begin{array}{l}\text { Density 2 } \\
\text { (sparse vegetation) }\end{array}$ & $\begin{array}{l}\text { Density 3 } \\
\text { (dense vegetation) }\end{array}$ \\
\cline { 2 - 4 } Cylinder $/ \mathrm{m}^{2}$ & 0 & 139 & 556 \\
$C_{d} \cdot a\left[\mathrm{~cm}^{-1}\right]$ & 0 & 0.014 & 0.056 \\
$Q_{\text {input }}\left[\mathrm{L} \mathrm{s}^{-1}\right]$ & $45,60,80$ & $45,60,80$ & $45,60,80$ \\
$H_{\text {control }}[\mathrm{cm}]$ & $12,13,14 \mathrm{~cm}$ & $12,13,14 \mathrm{~cm}$ & $12,13,14 \mathrm{~cm}$ \\
Floodplain Width $[\mathrm{m}]$ & $0,5 \mathrm{~m}, 0.25 \mathrm{~m}, 0.1 \mathrm{~m}$ & $0,5 \mathrm{~m}, 0.25 \mathrm{~m}, 0.1 \mathrm{~m}$ & $0.5 \mathrm{~m}, 0.25 \mathrm{~m}, 0.1 \mathrm{~m}$ \\
\hline
\end{tabular}

\section{Results}

The experiments have been performed for scenarios with different discharges $(Q)$, water depths $(H)$ and floodplain width $(W)$ (Table 1$)$. The presence of the vegetation, LHCSs and their effects on the shallow flow field are the major focus of this paper.

\subsection{Vegetation and Large Horizontal coherent structures}

The presence of vegetation and associated LHCSs is the main reason for the differences in the profile of the mean streamwise velocity and mixing layer. In the case without vegetation ( $0.5 \mathrm{~m}$ floodplain width), the mean streamwise velocity gradually reduce over the transition slope region from the main channel towards the floodplain. Transversely uniform values are reached at the edge of the floodplain meaning that the velocity profiles in this region are fully developed. In the cases of a vegetated floodplain channel, due to the presence of cylinders, the magnitude of the velocity gradient increases about 4 times. The mean streamwise velocity slowly reduces from the main channel before a sudden significant decrease at the middle of the transition slope. Furthermore, the mixing layer penetrates about $0.1 \mathrm{~m}$ into the vegetation areas.

The large coherent vortices can be recognized through the power density spectra (PDSs). The low-frequency side of the PDSs has large peaks with slope of approximately -3 . This indicates the presence large coherent structure with $2 \mathrm{D}$ characteristics [8]. These peaks in the 


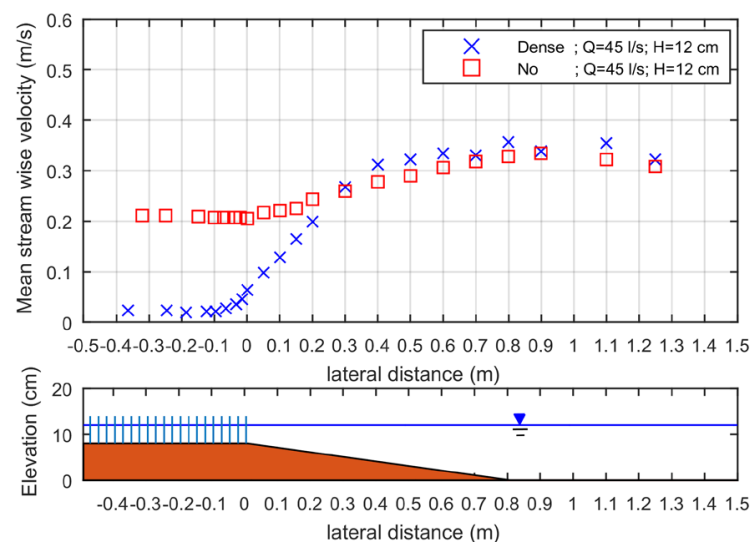

Figure 3. The effects of vegetation on the mean streamwise velocity and Reynold shear stresses of vegetated compound channel; $Q=45 \mathrm{~L} \mathrm{~s}^{-1}, H=12 \mathrm{~cm}$

PDS are at a frequency in the range of $0.05 \mathrm{~Hz}$ to $0.1 \mathrm{~Hz}$ corresponding to an eddy period of $10 \mathrm{~s}$ to $20 \mathrm{~s}$. The peaks have virtually disappeared at location $y=1.1 \mathrm{~m}$ (Figure 4a) and in the main channel(Figure $4 \mathrm{~b}$ ). This implies the disappearance of the LHCSs at that position. The decrease in energy density at the locations outside the floodplain region $(1.1 \mathrm{~m})$ almost follows a slope $-5 / 3$ which is indicative of flows with a large inertial subrange. However, inside the floodplain region $(y=0 m)$, the decrease of the energy density does not properly follow this rule. This is possibly due to the effect of the vortex shedding associated with the wakes behind the stems.

A pattern of the LHCSs can also be determined through the auto correlation function (Rvv) of the fluctuating transverse velocities. The Rvv of the fluctuating transverse velocities can be determined according to the equation (1), in which the brackets denote aeraging over all values of $t_{0}$.

$$
R_{v v}(t)=\frac{\left\langle v\left(t_{0}\right) \cdot v\left(t_{0}+t\right)\right\rangle}{\sqrt{\left\langle v^{2}\left(t_{0}\right)\right\rangle\left\langle v^{2}\left(t_{0}+t\right)\right\rangle}}
$$

The representative profiles of the Rvv at the edge of the floodplain and at different locations, in case with vegetation, are shown in Figure $4 \mathrm{c}$ and d, respectively. It can be seen that the correlation falls off slowly and its amplitude extrapolated to $t=0$ indicates that the LHCSs contribute about $50 \%$ to the total turbulent kinetic energy (at the edge, $y=0 \mathrm{~m}$ ). The modulation of the auto-correlation function beyond this point is related to the effects of the LHCSs [8]. The time scales of the small and large scale turbulences are of the order of about 1 second and 15 second, respectively. Further away from the mixing layer, the modulation of the autocorrelation function is smaller and the decrease of the autocorrelation function is faster. It can be seen that the LHCSs are more pronounced in cases with vegetation as the modulation of the autocorrelation function in cases with vegetation is significantly stronger than in cases without vegetation. The strong modulation of the auto-correlation function can not be seen outsite the mixing layer in the main channel Figure $4 \mathrm{~d}$. This indicates that there are no LHCSs at those locations. 

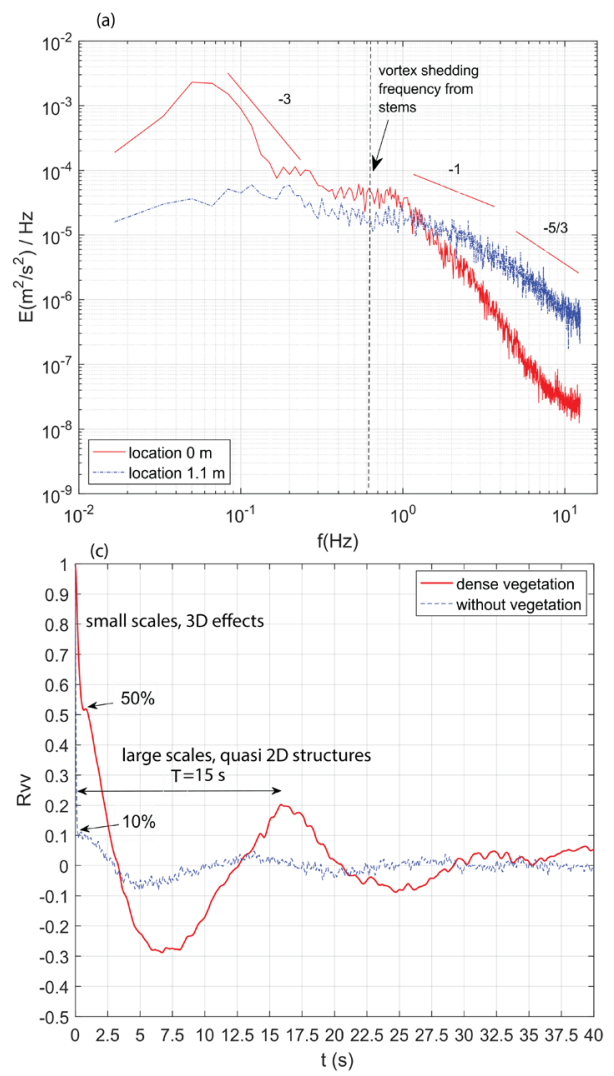

(b)

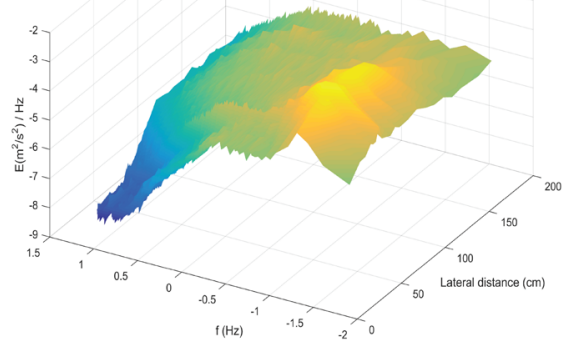

(d)

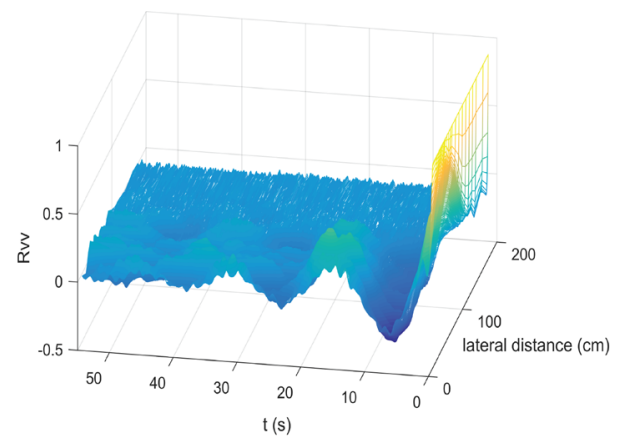

Figure 4. (a) Power density spectra (PDSs) of lateral velocity fluctuations at different locations: $y=0 \mathrm{~m}$ located at the vegetated floodplain edge, and $y=1.1 \mathrm{~m}$ located in the main channel ; (b) PDSs along the cross-section of the flume determined by mid-depth measurement at 22 locations; (c) Autocorrelation (Rvv) of fluctuating transverse velocities at the vegetation interface in cases with and without vegetation; (d) Rvv along the cross-section of the flume (mid-depth measurement at 22 locations) in the associated scenarios: dense scenario, $50 \mathrm{~cm}$ width, discharge $Q=45 \mathrm{~L} \mathrm{~s}^{-1}$, water level $H=12 \mathrm{~cm}$.

\subsection{Shallow flow fields}

The representative profile of the mean streamwise velocity measured by ADV can be seen inFigure 5. The flow field of the vegetated floodplain channel can be divided into three regions under the effects of LHCSs: the uniform region inside the floodplain (region I), the stable region outside in the main channel (region II), and the mixing layer region (region III and IV). The main factor in the mixing layer controlling the transverse momentum exchange between adjacent regions are the LHCSs. As the LHCSs move along the vegetated floodplain, they generated flow events which contribute a major part to the transverse momentum exchange between the open channel region and the vegetation region. This exchange has unquestionably ecological implications as it plays an important role in the transport of nutrients and sediment toward the forest area.

As a LHCS moves, it creates inflows that may be able to penetrate into the low flow region, termed sweeps $\left(u^{\prime}>0, v^{\prime}<0\right.$, where $u^{\prime}$ and $v^{\prime}$ are fluctuating streamwise and transverse velocities, respectively). This event, is then followed by outflows, termed ejections 


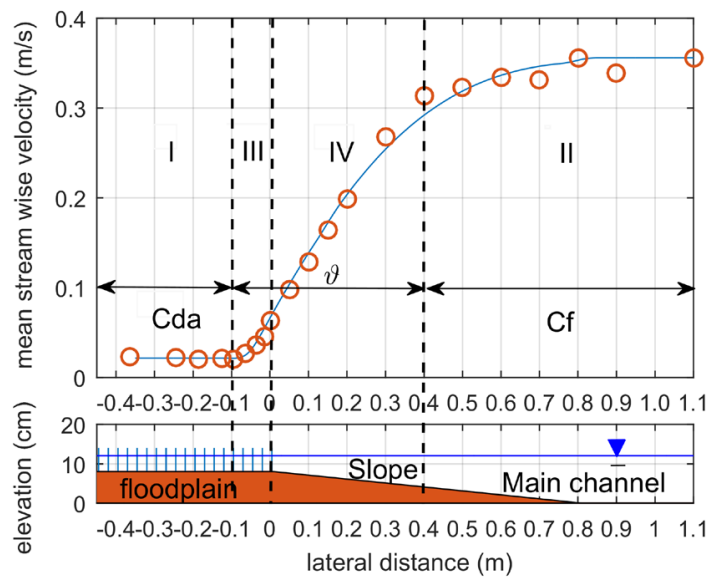

Figure 5. Representative profile of the compound channel (used in the experiment) and its corresponding representative time, depth and spatial averaged streamwise velocity under the effect of the LHCSs. Dense scenario, $50 \mathrm{~cm}$ floodplain width, discharge $Q=45 \mathrm{~L} \mathrm{~s}^{-1}$, water depth $D_{c}=12 \mathrm{~cm}$. The vegetated floodplain starts from $y=0 \mathrm{~cm}$ and in the negative direction.

$\left(u^{\prime}<0, v^{\prime}>0\right)$. The moving coherent structures also significantly disturb the slow flow field inside cylinder arrays. These cyclic flow events, created by the large coherent structures, can be observed to be the ones dominating the whole flow field of the floodplain region, especially in cases with vegetation.
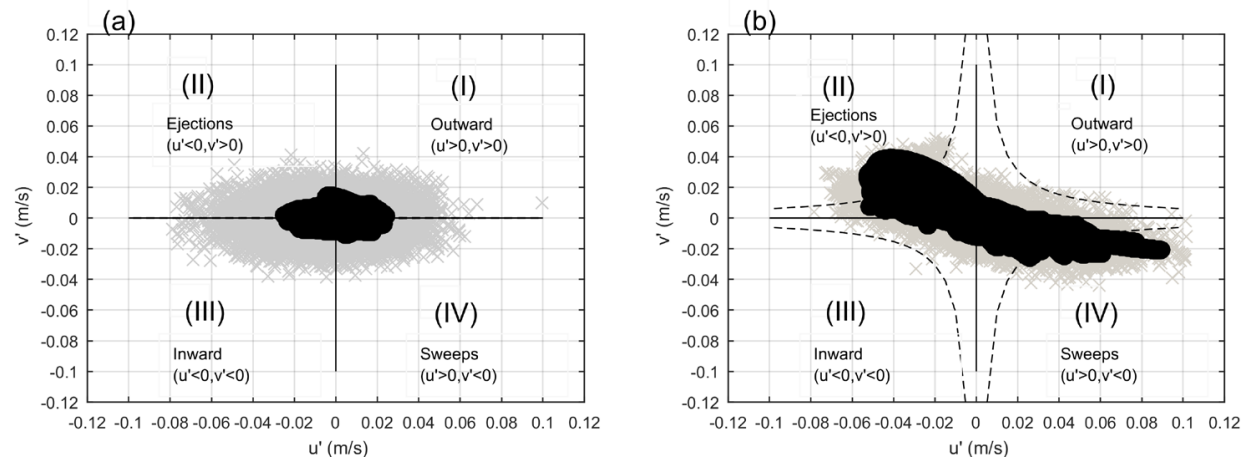

Figure 6. The quadrant distribution of the Reynolds shear stresses at the edge of floodplain $(y=0 \mathrm{~m})$, the associated low-frequency signal distribution (circles-darker) and the measure signal (cross-grey) in cases of no vegetation (a) and dense vegetation (b), $50 \mathrm{~cm}$ width, discharge $Q=45 \mathrm{~L} \mathrm{~s}^{-1}$, water level $H=12 \mathrm{~cm}$.

Quadrant analysis of the Reynolds shear stress allows to obtain useful information on the contribution to the total turbulence production from various events in the flows $[9,10]$. According to the signs of u' and v', quadrant analysis divides the Reynolds shear stresses into four quadrants (I-Q1, II-Q2, III-Q3, IV-Q4), which are associated with four different events of flow: outward interactions $\left(u^{\prime}<0, v^{\prime}>0\right)$, ejections $\left(u^{\prime}>0, v^{\prime}>0\right)$, inward interactions $\left(u^{\prime}<0, v^{\prime}<0\right)$ and sweeps $\left(u^{\prime}>0, v^{\prime}<0\right)$. A quadrant analysis of the Reynolds shear 
stresses $\left(-\overline{u^{\prime} v^{\prime}}\right)$ including the filtered low-frequency signals from "large" quasi $2 \mathrm{D}$ coherent structures (darker-black circles) which correspond to the LHCSs, at the edge of floodplain $(y=0 m)$ with and without vegetation can be seen in Figure 6a and b. In the case without vegetation, the LHCSs are less pronounced and the distribution of ( $\left.\mathrm{u}^{\prime}, \mathrm{v}^{\prime}\right)$ corresponding to the "large" structures is relatively equally distributed in Q1, Q2, Q3 and Q4. Hence, the contribution of the Reynolds shear stresses to the total turbulence production is small. However, in the case with vegetation, the results suggested that the distribution of (u', v') corresponding to the "large" structures is mainly distributed in Q2 and Q4, which are related to the ejection and sweep events. This result is in line with the observations in the partly vegetated channel of $[6,7]$. It also shows that LHCSs, through sweep and ejection events contribute a major part to the transverse momentum exchange between the main channel and the vegetation region.

\section{Discussions}

Following the dye tracer, the flow field under the effects of LHCSs can be observed. The observation suggests that the tracer path of the dye does follow a cycloid curve (Figure 7). The term "cycloid" adopted in our study was initiated from the observations and analyses of the instantaneous flow velocity field captured by the PIV measurement [11]. As a LHCS moves, part of it moves inside the vegetated floodplain (the penetration part) and part of it moves in the adjacent open channel. Inside the vegetation, the stagnant $(u \approx 0, v \approx 0)$ and reverse flows $(u<0)$ can be observed (see Figure 8$)$. These phenomena are the traces left by the LHCSs as they move along the vegetation interface and appear to be a proof for this peculiar characteristic of the flow field [11].
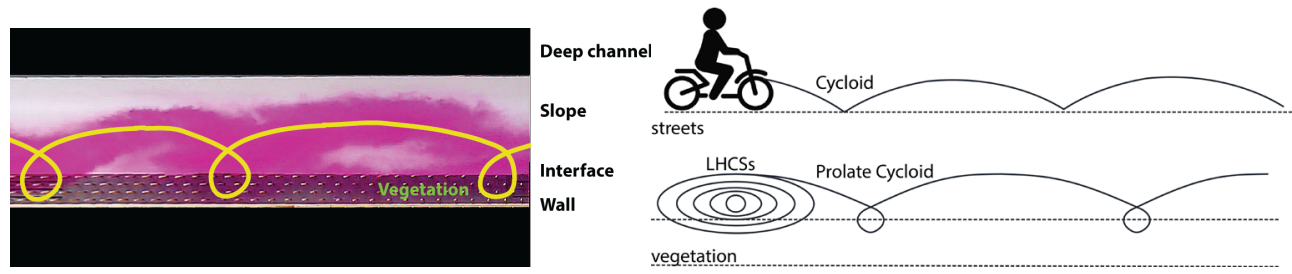

Figure 7. The flow field under the effects of LHCSs captured by the dye tracer (with sketched trajectory) has a cycloid characteristic; Sparse scenario : $25 \mathrm{~cm}$ width, discharge $Q=45 \mathrm{~L} \mathrm{~s}^{-1}$ and water level $H=12 \mathrm{~cm}$.

\section{Conclusions}

The paper introduces initial results of the experiment of a compound vegetated channel. The results confirm the very strong presence of the large coherent structures and their effects on the hydrodynamic of the vegetated floodplain channel. The presence of floodplain and vegetation creates a gradient in the flow velocity between the floodplain region and the adjacent open channel, leading to the generation of large coherent structures in the mixing layer. As the large coherent structure moves along the interface between cylinder arrays and the open channel, it penetrates into and disturbs the slow flow region inside the forest. As a result, cycloid flow events induced by the LHCSs dominate the flow field of the vegetated compound channel and contribute a large part to the total transverse exchange of mass and momentum. Future work will focus on a further interpretation of the observed phenomena and a proper numerical modelling of the exchange processes. 


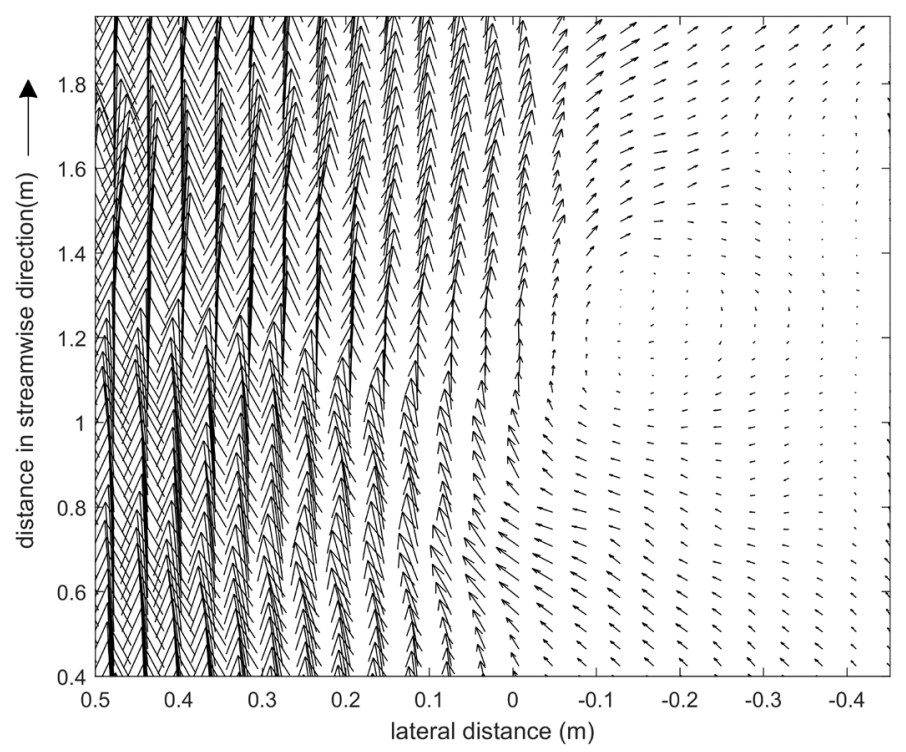

Figure 8. Instantaneous velocity field captured by PIV measurement. Dense scenario, $50 \mathrm{~cm}$ floodplain width, discharge $Q=45 \mathrm{~L} \mathrm{~s}^{-1}$, water depth $D_{c}=12 \mathrm{~cm}$. The vegetated floodplain starts from $y=0 \mathrm{~cm}$ and in the negative direction.

\section{References}

[1] L.K. Phan, J.S. van Thiel de Vries, M.J. Stive, Journal of Coastal Research 300, 233 (2015)

[2] S.H. Truong, Q. Ye, M.J. Stive, Journal of Coastal Research pp. 747-763 (2017), https://doi .org/10.2112/JCOASTRES-D-16-00087.1

[3] N. Tamai, T. Asaeda, H. Ikeda, Water Resources Research 22, 1129 (1986)

[4] I. Nezu, K. Onitsuka, Journal of Hydraulic Research 39, 629 (2001)

[5] B. van Prooijen, B.J. A., U.W.S. J., Journal of Hydraulic Engineering 131, 175 (2005)

[6] B.L. White, H.M. Nepf, Journal of Fluid Mechanics 593, 1 (2007)

[7] B.L. White, H.M. Nepf, Water Resources Research 44 (2008)

[8] W. Uijttewaal, R. Booij, Physics of fluids 12, 392 (2000)

[9] W. Willmarth, S. Lu, Journal of Fluid Mechanics 55, 65 (1972)

[10] J. Kim, P. Moin, R. Moser, Journal of fluid mechanics 177, 133 (1987)

[11] H. Truong, W. Uijtewaal, M. Stive, Water Resources Rearch (under review) (2018) 